\title{
Small and Robotic Telescopes in the Era of Massive Time-Domain Surveys
}

\author{
M. F. Bode ${ }^{1}$ and W. T. Vestrand ${ }^{2}$ \\ ${ }^{1}$ Astrophysics Research Institute, Liverpool JMU, Birkenhead, CH41 1LD, UK \\ email: mfb@astro.livjm.ac.uk \\ ${ }^{2}$ Center for Space Science and Exploration, Los Alamos National Laboratory, \\ NM 87545, USA
}

\begin{abstract}
We have entered an era in time-domain astronomy in which the detected rate of explosive transients and important ephemeral states in persistent objects threatens to overwhelm the world's supply of traditional follow-up telescopes. As new, comprehensive time-domain surveys become operational and wide-field multi-messenger observatories come on-line, that problem will become more acute. The goal of this workshop was to foster discussion about how autonomous robotic telescopes and small-aperture conventional telescopes can be employed in the most effective ways to help deal with the coming deluge of scientifically interesting follow-up opportunities. Discussion topics included the role of event brokers, automated event triage, the establishment of cooperative global telescope networks, and real-time coordination of observations at geographically diverse sites. It therefore included brief overviews of the current diverse landscape of telescopes and their interactions, and also considered planned and potential new facilities and operating models.
\end{abstract}

\section{Robotic Telescopes}

Iain Steele (Liverpool JMU) gave a brief presentation on "Robotic Telescopes". A robotic telescope is one that can operate autonomously, that is, without night-time supervision. It is important to remember that small and robotic are not synonymous. There are many smaller telescopes that are not robotic, and several larger telescopes that are. It is generally assumed that such facilities are relevant only to the optical/NIR, and that was certainly the focus of this session. It would be interesting to consider what contribution could be made at other wavelengths (especially the radio) by such facilities. Can we rely just on facilities such as LOFAR and the SKA precursors to monitor and follow-up the time-domain radio sky?

For the optical/NIR, Hessman provides a very useful webpage $†$ where he lists a whole raft of robotic-telescope projects. At the time of writing the list contained 122 projects, of which around $17 \%$ are general-purpose facilities and the remainder are dedicated to one or two science goals such as surveys for exoplanets or Gamma-Ray Burst followups. Those in the latter class are usually run as "experiments", which can result in considerable cost savings compared to a common-user robot.

Another advantage of experiments can be the optimisation of telescope and instrument design for the science goal. As an example, consider the question of "small and quick" versus "large and slow" for the follow-up of transient sources. For Gamma-Ray Bursts, the photon rate in the afterglow phase scales as $t^{-1}$. Experience shows that the typical slew time of a telescope to a source scales roughly as the mirror diameter, $D$. The photon rate will therefore be proportional to $D^{-1}$, and since detection area scales as $D^{2}$

$\dagger$ http://www. uni-sw.gwdg.de/ hessman/MONET/links.html 
the number of photons detected will be roughly proportional to $D$. In this particular case, the advantage of aperture therefore wins over that of speed. Similar calculations can be made for other experiments too, and show (for example) that for survey work the advantage of larger sky area versus depth can favour smaller facilities.

An important question that we should address relates to the balance of instrumentation available on robotic facilities. In general robotic telescopes are equipped with CCD imagers, and very few have NIR cameras or spectrographs and even fewer have polarimetric facilities. Is this balance the correct one in an era when LSST and other large sky surveys will provide regular photometric monitoring? Whatever instrumentation is used, however, it is very important that it be supported by some sort of data-reduction pipeline which can reduce the data to a level sufficient for reliable use in planning and triggering further observations in a timely fashion. This latter point was returned to later by Vestrand (Section 4).

Some of the major "feeder" facilities of the future are not common-user ones, and preparations need to be made well ahead of time to ensure that the necessary follow-up agreements are in place.

\section{The AAVSO Perspective}

Arne Henden (AAVSO) emphasised the role of the amateur community in this endeavour, and spoke particularly about the various networks that now exist. The AAVSO has established a global network of 19 robotic telescopes, from $60-\mathrm{mm}$ to 80 -cm aperture. The telescopes are heterogenous, but the software is not. All the images are pipeline-processed at AAVSO HQ, and images are transferred to Amazon Cloud for users to access. Work is under way to build a master scheduler based on VOEvent.

The challenges faced by the network include continuity and longevity of private sites, internet access at remote locations, data archiving and access to that archive, maintenance, problems typical of a "volunteer" situation, time allocation, and the presence of non-imaging instruments (spectrographs) at two of the larger telescopes which add to the potential science return but also add to the complexity. Several of those issues are of course common to professional networks too (see Section 4).

\section{Follow-up of Gaia Alerts}

Łukasz Wyrzykowski (Cambridge) focussed on GAIA, ESA's milestone mission which is to be launched in 2013 June. The mission's main goal is to map the Galaxy with ultra-precise astrometry and photometry of a billion stars, but owing to the repeated nature of the observations GAIA will also be very sensitive to anomalous and transient events. An alert will normally be issued during an interval of $2-24$ hours after the detection of such an event. The depth of the survey is about $V=20$ mag. The sampling is sparse, yielding on average 80 observations per object over the 5 -year mission. Anomaly detection will therefore be based on as few as 1 or 2 GAIA data points, which will not be enough to characterise fully the detected phenomena or to exploit maximally the science involved. GAIA therefore requires an extensive ground-based follow-up capability for the verification and classification of the alerts that it reports. With hundreds to thousands of alerts expected every day, the situation calls for a network of robotised telescopes sited all around the globe. 
The community is proposing to organise a formal consortium of telescopes and observers under the auspices of the European OPTICON network $\dagger$, primarily with GAIA alerts in mind but also more generally for following up all sorts of time-critical cosmic events. The network is currently being assembled in the wake of two GaIA Science Alerts Workshops $\ddagger$ held in Cambridge, UK. The network will be comprised of small $(<2-\mathrm{m})$ telescopes, mostly robotised, which are able to provide both multi-band imaging and lowto high-dispersion spectroscopy. The observational outputs will need to be standardised. There will also need to be clear rules on data policy, publishing, etc.

\section{Factors to Consider for Effective Robotic Telescope Networks}

Ashish Mahabal (Caltech) gave a brief presentation in which he emphasised that following up transient sources is complex for various scientific and sociological reasons. Different telescopes can observe only a subset of possible transients because (1) their aperture limits it, (2) the available instruments determine it, (3) the interests of the people running the telescope interfere with it (potentially) - plus a host of other reasons like weather, RA/Dec coverage, and (most importantly) the state of preparedness (robotic versus automated versus semi-automated versus manual).

The problem is going to get far worse as more transients are found, a majority of which (it will turn out after some observations) are of a relatively common nature (i.e. we already know a lot about their classes). It will therefore be critical to make early classification with minimal data in order to determine which transients are in fact most worthy of following up. One helpful procedure will be to obtain priors of different telescope/instrument combinations so that one might decide how likely it will be for an observation from a given instrument set-up to disambiguate the possible classes to which the transient might belong.

Another crucial factor is prompt feedback from the observatories. It is not enough for the observatories just to receive events: without feedback, further classification and informed follow-up will not be possible. Those stages also need a degree of automated pipeline processing.

Some work on these issues is already underway at Caltech, and in collaboration with the GaIA consortium, in particular the Gaia Alerts group at Cambridge.

Tom Vestrand (LANL) began by stating some of the assumed characteristics that went into developing an effective network of robotic telescopes for real-time follow-up of unpredictable events. They included real-time inter-communication between telescopes, heterogeneous instrumentation and general capabilities, and free exchange of information and knowledge between systems. It was felt that in the future there could ultimately be more robotic instruments than astronomers, with each telescope playing a dynamic role.

Currently however, the way we handle time-domain events is very much like the "second-grade soccer" problem - exciting, enthusiastic, but disorganised. An effective network would be "self-aware" - knowing what tasks each telescope is doing now, what the state of health of each system is in terms of instrument configuration, general capability, etc., what are the sky conditions at the telescope's location and whether it can observe effectively at the target location.

Locally, each telescope's "decision engine" would have to answer questions such as whether it has the sensitivity to make the required observation, what is the relative importance of the observation compared to what it is currently doing, how well it matches

$\dagger$ http://www.astro-opticon.org/

$\ddagger$ http://www.ast.cam.ac.uk/ioa/wikis/gsawgwiki/index.php/Workshop2011:main 
the telescope's "uniqueness" parameters, what the other network nodes are doing, and what is the highest-impact observation that the telescope can make at that moment.

In terms of what needs to be done, it is important to all follow-up projects, particularly with such a prospective deluge of alerts likely to be seen in the coming years, that event classification be as automated and reliable as possible. Similarly, event brokers need to become fully developed. Real-time feedback is also going to be critical, in the sense of knowing what is being found at each instant and then determining what the next step should be. Finally, "trust models" for all the sources of information being fed into and among the network components need to be developed.

To summarise: we are entering an era where a global ecosystem of robotic telescopes could make unpredicted measurements and discoveries in time-domain astronomy. Most of the technical elements are available or under development. The biggest problem is likely to be the sociology of humans.

\section{Summary and Conclusions}

- It is evident that in the era of massive time-domain surveys there is an increasingly important role for telescopes of modest aperture, but the required reaction speeds demand that they be robots.

- For effective and efficient responses for single telescopes, but particularly for networks, we need to embrace a "human out of the loop" philosophy - this includes almost every facet of automated data reduction.

- Amateurs can make important contributions in this regime with their relatively small telescopes but increasingly high levels of sophistication.

- Alert characterisation is crucial. For example, in the coming era, what not to observe will be a central theme.

- For robotic telescope networks, a full understanding of the roles of individual instruments is needed, plus the development of "trust models" and real-time "state of health" detection.

- The balance of sites, apertures and types of instrumentation needs to be considered carefully as it is likely to be sub-optimal for future scientific demands.

- The network needs to have efficient ways to interrogate its components in real time, and dynamic ways to determine "what next".

- Similarly, there is a great deal of work to be done on the integration of telescope ecosystems with well-defined roles for individual instruments.

- In some instances agreements will need to be struck with upcoming feeder facilities well ahead of their operational debuts, but overall, optimising the scientific return in the future will depend on developing focused science experiments. 